\title{
Fermion Propagators in Type II Fivebrane Backgrounds
}

\author{
Noriaki Kitazawd* \\ Department of Physics, Tokyo Metropolitan University, Hachioji, Tokyo 192-0397, Japan
}

(Dated: November 14, 2018)

\begin{abstract}
The fermion propagators in the fivebrane background of type II superstring theories are calculated. The propagator can be obtained by explicitly evaluating the transition amplitude between two specific NS-R boundary states by the propagator operator in the non-trivial world-sheet conformal field theory for the fivebrane background. The propagator in the field theory limit can be obtained by using point boundary states. We can explicitly investigate the lowest lying fermion states propagating in the non-trivial ten-dimensional space-time of the fivebrane background: $M^{6} \times W_{k}^{(4)}$, where $W_{k}^{(4)}$ is the group manifold of $\mathrm{SU}(2)_{k} \times \mathrm{U}(1)$. The half of the original supersymmetry is spontaneously broken, and the space-time Lorentz symmetry $\operatorname{SO}(9,1)$ reduces to $\mathrm{SO}(5,1)$ in $\mathrm{SO}(5,1) \times \mathrm{SO}(4) \subset \mathrm{SO}(9,1)$ by the fivebrane background. We find that there are no propagations of $\mathrm{SO}(4)$ (local Lorentz) spinor fields, which is consistent with the arguments on the fermion zero-modes in the fivebrane background of low-energy type II supergravity theories.
\end{abstract}

*Electronic address: kitazawa@phys.metro-u.ac.jp 


\section{INTRODUCTION}

The quantum effect of gravity may shed new light on the unsolved problems in particle physics. It was pointed out that supersymmetry can be spontaneously and dynamically broken by the gravitino condensation [1, 2] in non-trivial space-time backgrounds. It was also suggested that all the global symmetries in the low-energy effective field theory should be broken by the effect of quantum gravity [3, 4]. It must be very important to investigate these possibilities in the string theory which is a strong candidate for the consistent theory of quantum gravity.

If we have a fermion propagator, or a fermion two point function, in some non-trivial backgrounds (space-time metric or gauge fields), we can obtain a value of the fermion pair condensate which can be an order parameter of some symmetry breaking. For example, in case of the $\mathrm{SU}(N)$ gauge theory with massless vector-like fermions in the fundamental representation of the gauge group in Euclidean space-time, the zero-instanton sector in the path integral of the fermion two-point function gives Euclidean propagator, and the oneinstanton sector gives the fermion pair condensate which triggers chiral symmetry breaking. In the same system in Minkowski space-time full propagator should include the mass function whose integral gives the value of the fermion pair condensate through the arguments of the operator product expansion. Therefore, it is interesting to investigate the fermion propagator in non-trivial space-time backgrounds in string theories.

The fivebrane background is a non-trivial background of space-time metric and fields in type IIA, type IIB and heterotic string theories [5, 6, 7]. The half of the original supersymmetry is broken, and the space-time Lorentz symmetry reduces to $\mathrm{SO}(5,1)$ from $\mathrm{SO}(9,1) \supset$ $\mathrm{SO}(5,1) \times \mathrm{SO}(4)$ by the fivebrane background. Although general fivebrane backgrounds are realized as the solutions in the low-energy supergravity theory of each string theory, in some special case they can be described by world-sheet conformal field theories as the solutions of string theories[8, 9]. Therefore, in principle we can calculate fermion propagators in string theories perturbatively with respect to the string coupling without any low-energy approximation [10, 11].

The number of fermion zero-modes in non-trivial four-dimensional space of fivebrane backgrounds in low-energy supergravity theories is well-known 7]. There are four fermion zero-modes in type II theories 12], and two fermion zero-modes in heterotic theories [13]. 
If we believe the path integral formalism of the supergravity theory, these numbers mean four fermion condensations in type II theories and fermion pair condensations in heterotic theories in non-trivial four-dimensional space. Although the case of the heterotic theory is much more interesting than the case of the type II theory in the strategy of extracting the value of the fermion pair condensate from the propagator, there are some technical difficulties to calculate the fermion propagator (especially for gravitino and dilatino) in heterotic string theories. (The calculation of the gaugino propagator is possible, and the work is underway.) In this paper we calculate the gravitino and dilatino propagator in the fivebrane background in type II string theories as the first attempt. If the path integral formalism of the supergravity theory is correct, there should be no propagations of the light fields in $\mathrm{SO}$ (4) (local Lorentz) spinor representations.

The paper is organized as follows. In the next section we give a brief review of the fivebrane background in type II string theories. The conformal field theory for the fivebrane background is introduced. In Sec $\amalg$ the closed string boundary state to which a single fermion state (NS-R state) can couple is introduced. In Sec IV fermion propagators are calculated by evaluating the transition amplitude between boundary states by the propagator operator in the world-sheet conformal field theory. In the last section we summarize our results and give some comments.

\section{FIVEBRANE BACKGROUNDS IN TYPE II STRING THEORIES}

The fivebrane background (or the NS5-brane) is a BPS configuration which preserves half of the supersymmetry of the theory. In type II supergravity theories, which are the low-energy effective theories of type IIA and type IIB string theories, the space-time metric configuration of the fivebrane background (NS5-branes at the origin) is explicitly given by

$$
\begin{aligned}
& g_{\mu \nu}=e^{2 \Phi} \delta_{\mu \nu}, \\
& e^{2 \Phi}=e^{2 \Phi_{0}}+\frac{n \alpha^{\prime}}{r^{2}},
\end{aligned}
$$

where $n$ is an integer, $\mu=6,7,8,9$ are the index of the non-trivial four-dimensional space

in the whole ten-dimensional space-time, and $r^{2}=\sum_{\mu=6}^{9} x^{\mu} x^{\mu}$. We use $\hat{\mu}=0,1, \cdots, 9$ as the index of the whole ten-dimensional space-time, and use $\bar{\mu}=0,1, \cdots, 5$ as the index

of the flat six-dimensional Minkowski space-time in the whole ten-dimensional space-time 
from now on. The geometry of the space-time is $M^{6} \times R \times S^{3}$ with varying radius of $S^{3}$ from $\sqrt{n \alpha^{\prime}}$ to infinity along with the value of the coordinate of $R$ from $-\infty$ to $\infty$. These solutions in low-energy supergravity theories are considered to be the exact solutions in type II string theories. The world-sheet conformal field theory for these backgrounds can be explicitly constructed in case of $e^{2 \Phi_{0}}=0$. In this case the space-time geometry is $M^{6} \times W_{k}^{(4)}$, where $W_{k}^{(4)}$ is the four-dimensional group manifold of $\mathrm{SU}(2)_{k} \times \mathrm{U}(1)$ with Kač-Moody level $k$. The geometry of $W_{k}^{(4)}$ is again $R \times S^{3}$, but the radius of $S^{3}$ is fixed with $\alpha^{\prime} / Q$, where $Q=\sqrt{\alpha^{\prime} /(k+2)}$.

In case of the flat space-time the world-sheet theory of the type II string theory consists of one free bosonic field $X^{\hat{\mu}}(z, \bar{z})$ and two free fermionic fields $\psi^{\hat{\mu}}(z)$ and $\tilde{\psi}^{\hat{\mu}}(\bar{z})$. The system has $(N, \tilde{N})=(1,1)$ superconformal symmetry with the central charge $c_{m}=\tilde{c}_{m}=10+10 / 2=15$ which is cancelled by the ghost contribution $c_{g}=\tilde{c}_{g}=-26+11=-15$. The non-trivial background $M^{6} \times W_{k}^{(4)}$ can be described by replacing unconstrained fields $X^{\mu}(\mu=6,7,8,9)$ with the fields constrained on the group manifold of $\mathrm{SU}(2)_{k} \times \mathrm{U}(1)$. Namely, the part of the world-sheet theory corresponding to the space-time coordinates of $\mu=6,7,8,9$ is replaced by the combination of the $\mathrm{SU}(2)_{k}$ WZW model and the linear-dilaton theory. The new part has $(N, \tilde{N})=(4,4)$ superconformal symmetry, and has the same central charge of the original part: $c_{4 D}=6$.

The holomorphic sector of the $\mathrm{SU}(2)_{k} \times \mathrm{U}(1)$ part is described by three $\mathrm{SU}(2)_{k}$ bosonic currents $J_{i}(z)(i=1,2,3)$, one free bosonic current $J_{4}(z)=\partial X^{\mu=6}$ and four free fermionic fields $\Psi_{a}(a=1,2,3,4)$. These currents and fields satisfy the following operator product expansion. (We set $\alpha^{\prime}=2$ from now on in this section for simplicity.)

$$
\begin{aligned}
J_{i}(z) J_{j}\left(z^{\prime}\right) & \sim-\frac{k}{2} \frac{\delta_{i j}}{\left(z-z^{\prime}\right)^{2}}+\epsilon_{i j l} \frac{J_{l}}{z-z^{\prime}}, \\
J_{4}(z) J_{4}\left(z^{\prime}\right) & \sim-\frac{1}{\left(z-z^{\prime}\right)^{2}}, \\
\Psi_{a}(z) \Psi_{b}\left(z^{\prime}\right) & \sim-\frac{\delta_{a b}}{z-z^{\prime}} .
\end{aligned}
$$

The $N=4$ superconformal symmetry transformation is generated by the following energymomentum tensor $T^{W_{k}^{(4)}}(z)$, supercurrents $G_{a}^{W_{k}^{(4)}}(z)$ and $\mathrm{SU}(2)_{n}$ currents $S_{i}(z)$.

$$
\begin{aligned}
T^{W_{k}^{(4)}} & =-\frac{1}{2}\left(\frac{2}{k+2} J_{i}^{2}+J_{4}^{2}-\Psi_{a} \partial \Psi_{a}+Q \partial J_{4}\right), \\
G_{i}^{W_{k}^{(4)}} & =\sqrt{\frac{2}{k+2}}\left(J_{i} \Psi_{4}-\epsilon_{i j l} J_{j} \Psi_{l}+\frac{1}{2} \epsilon_{i j l} \Psi_{4} \Psi_{j} \Psi_{l}\right)-J_{4} \Psi_{i}-Q \partial \Psi_{i},
\end{aligned}
$$




$$
\begin{aligned}
G_{4}^{W_{k}^{(4)}} & =\sqrt{\frac{2}{k+2}}\left(J_{i} \Psi_{i}+\frac{1}{3 !} \epsilon_{i j l} \Psi_{i} \Psi_{j} \Psi_{l}\right)+J_{4} \Psi_{4}+Q \partial \Psi_{4}, \\
S_{i} & =\frac{1}{2}\left(\Psi_{4} \Psi_{i}+\frac{1}{2} \epsilon_{i j l} \Psi_{j} \Psi_{l}\right) .
\end{aligned}
$$

The background charge $Q$ determines the gradient of the linear-dilaton background $\Phi=$ $Q X^{\mu=6}$. The world-sheet field $X^{\mu=6}(z, \bar{z})$ is called the Feigin-Fuchs field, and the theory of this field is called the linear-dilaton theory. The value $Q=\sqrt{\alpha^{\prime} /(k+2)}=\sqrt{2 /(k+2)}$ is required for the correct central charge of $c_{4 D}=6$, and the Kač-Moody level $n$ of $\mathrm{SU}(2)_{n}$ is fixed to unity due to the relation of $n=c_{4 D} / 6$. The anti-holomorphic sector has exactly the same structure.

The world-sheet theory of the $M^{6}$ part consists of one free bosonic field $X^{\bar{\mu}}(z, \bar{z})$ and two free fermionic fields $\Psi^{\bar{\mu}}(z)$ and $\bar{\Psi}^{\bar{\mu}}(\bar{z})$. In the holomorphic sector the energy-momentum tenor $T^{M^{6}}(z)$ and the supercurrent $G^{M^{6}}(z)$ are

$$
\begin{aligned}
T^{M^{6}} & =-\frac{1}{2} \partial X^{\bar{\mu}} \partial X_{\bar{\mu}}+\frac{1}{2} \Psi^{\bar{\mu}} \partial \Psi_{\bar{\mu}}, \\
G^{M^{6}} & =\Psi^{\bar{\mu}} \partial X_{\bar{\mu}} .
\end{aligned}
$$

The anti-holomorphic sector has exactly the same structure, and the $M^{6}$ part has $(N, \tilde{N})=$ $(1,1)$ superconformal symmetry. The whole world-sheet theory has $(N, \tilde{N})=(1,1)$ superconformal symmetry, and we take $T=T^{M^{6}}+T^{W_{k}^{(4)}}$ and $G=G^{M^{6}}+G_{4}^{W_{k}^{(4)}}$ as the currents of that symmetry. The super-Virasoro generators $L_{n}(n \in \mathbf{Z})$ and $G_{r}(r \in \mathbf{Z}+1 / 2$ in Neveu-Schwarz sector and $r \in \mathbf{Z}$ in Ramond sector) are defined by

$$
\begin{aligned}
L_{n} & =\oint \frac{d z}{2 \pi i z} z^{n+2} T(z), \\
G_{r} & =\oint \frac{d z}{2 \pi i z} z^{r+3 / 2} G(z) .
\end{aligned}
$$

The anti-holomorphic sector has exactly the same structure. The partition function, or one-loop vacuum amplitude, is explicitly calculated in Ref.[9] for even $k$. We consider $k$ as an even number from now on. The difference between type IIA and type IIB theories is the difference of the usual universal GSO projection. In addition to the universal GSO projection, we have to do the additional GSO projection by which the half breaking of the space-time supersymmetry is realized in the world-sheet theory. 


\section{BOUNDARY STATES FOR PROPAGATORS}

The propagator of the closed string in type II theories can be calculated as the transition amplitude between two appropriate boundary states by the propagator operator in the worldsheet conformal field theory. In case of the flat space-time, the propagator of bosonic states (in the bosonic string theory) is extensively studied in Ref.[14], and the propagators in the superstring theory are calculated in Refs. 10, 11]. In order to obtain the propagator in the field theory limit (not the low-energy limit), the point boundary states, which describe the states of the closed string shrinking to a point in space-time, should be used. For the bosonic (NS-NS or R-R) states of strings, we can use D(-1)-brane, or D-instanton, states [15]:

$$
|B(y)\rangle=\left|B_{X}(y)\right\rangle \otimes\left|B_{\psi}\right\rangle \otimes\left|B_{\mathrm{gh}}\right\rangle \otimes\left|B_{\mathrm{sgh}}\right\rangle
$$

where

$$
\left|B_{X}(y)\right\rangle=\delta^{10}(\hat{x}-y) \exp \left\{\sum_{n=1}^{\infty} \frac{1}{n} \alpha_{-n}^{\hat{\mu}} \tilde{\alpha}_{-n \hat{\mu}}\right\}|0\rangle
$$

describes the state of the closed string shrinking at a space-time point of $y$ (or the state where the edge of the open string is fixed at a space-time point of $y),\left|B_{\psi}\right\rangle$ is the worldsheet fermion contribution which is determined by the supersymmetry and T-duality, and $\left|B_{\mathrm{gh}}\right\rangle \otimes\left|B_{\mathrm{sgh}}\right\rangle$ is the contribution of world-sheet ghost fields, the $b c$-ghost state and the $\beta \gamma$-ghost state, respectively. (We are using the notation of Ref.[16] except for a factor $i$ for world-sheet fermion fields.) The propagator

$$
P_{B}\left(y^{\prime}-y\right)=\left\langle B\left(y^{\prime}\right)\left|D_{B}\right| B(y)\right\rangle
$$

with the propagator operator

$$
D_{B}=\frac{\alpha^{\prime}}{4 \pi} \cdot \frac{1}{2} \int_{|z| \leq 1} d^{2} z \frac{1}{|z|^{2}} z^{L_{0}} \bar{z}^{\tilde{L}_{0}}
$$

can be explicitly calculated.

For the fermionic (NS-R or R-NS) states of strings, we can not use the D-instanton states, because they do not couple with a single fermionic state. Therefore, we have to introduce some source boundary states which couple with a single fermionic state. The source states do not necessarily correspond to some physical objects like D-branes. We concretely introduce the following boundary state for NS-R fermionic states.

$$
|B(y, \mathbf{s})\rangle^{\hat{\mu}}=\left|B_{X}(y)\right\rangle \otimes\left|B_{\text {gh }}\right\rangle \otimes \psi_{-1 / 2}^{\hat{\mu}}|0\rangle_{\mathrm{NS}} \otimes \widetilde{\left|\psi_{\mathbf{s}}\right\rangle_{\mathrm{R}}}
$$


where $|0\rangle_{\mathrm{NS}}$ is the Neveu-Schwarz vacuum state and $\widetilde{\left|\psi_{\mathrm{s}}\right\rangle_{\mathrm{R}}}$ is the Ramond vacuum state. The spin state $\mathbf{s}=s_{1} \otimes s_{2} \otimes s_{3} \otimes s_{4} \otimes s_{5}$ with $s_{i}= \pm 1 / 2$ for $i=1,2, \cdots, 5$ is described by

$$
\psi_{\mathbf{s}}=\eta_{1} \otimes \eta_{2} \otimes \eta_{3} \otimes \eta_{4} \otimes \eta_{5}
$$

with

$$
\eta_{i}=\left(\begin{array}{l}
1 \\
0
\end{array}\right),\left(\begin{array}{l}
0 \\
1
\end{array}\right)
$$

for $s_{i}=1 / 2,-1 / 2$, respectively. The $b c$-ghost state $\left|B_{\mathrm{gh}}\right\rangle$ is explicitly given by

$$
\left|B_{\text {gh }}\right\rangle=\exp \left\{\sum_{n=1}^{\infty}\left(c_{-n} \tilde{b}_{-n}-b_{-n} \tilde{c}_{-n}\right)\right\} \frac{c_{0}+\tilde{c}_{0}}{2}|\downarrow\rangle \otimes|\widetilde{\downarrow}\rangle
$$

and

$$
\left\langle B_{\text {gh }}|=\widetilde{\langle\uparrow}| \otimes\langle\uparrow| \frac{\tilde{b}_{0}-b_{0}}{2} \exp \left\{\sum_{n=1}^{\infty}\left(\tilde{b}_{n} c_{n}-\tilde{c}_{n} b_{n}\right)\right\} .\right.
$$

Using the propagator operator

$$
D_{F}=\frac{\alpha^{\prime}}{4 \pi} \cdot \tilde{G}_{0} \cdot \frac{1}{2} \int_{|z| \leq 1} d^{2} z \frac{1}{|z|^{2}} z^{L_{0}} \bar{z}^{\tilde{L}_{0}}
$$

we can explicitly calculate the fermion propagator as follows.

$$
\begin{aligned}
P_{F}\left(y^{\prime}-y\right)_{\mathbf{s}^{\prime} \mathbf{s}}^{\hat{\mu} \hat{\nu}} & ={ }^{\hat{\mu}}\left\langle B\left(y^{\prime}, \mathbf{s}^{\prime}\right)\left|D_{F}\right| B(y, \mathbf{s})\right\rangle^{\hat{\nu}} \\
& =-i \frac{\sqrt{\alpha^{\prime}}}{2} \sum_{N_{X}=0}^{\infty} d\left(N_{X}\right) \cdot \int \frac{d^{10} q}{(2 \pi)^{10}} \bar{\psi}_{\mathbf{s}^{\prime}} q_{\hat{\rho}} \Gamma^{\hat{\rho}} \psi_{\mathbf{s}} \eta^{\hat{\mu} \hat{\nu}} \frac{1}{q^{2}+M_{N_{X}}^{2}} e^{i q_{\hat{\sigma}}\left(y^{\prime}-y\right)^{\hat{\sigma}}}
\end{aligned}
$$

where $M_{N_{X}}^{2}=4 N_{X} / \alpha^{\prime}$ with the level $N_{X}$ of the bosonic excitations on the world-sheet, and $d\left(N_{X}\right)$ is the degeneracy of the open superstring state with the bosonic level $N_{X}$ and zero fermionic level. In the momentum space

$$
P_{F}(q)_{\mathbf{s}^{\prime} \mathbf{s}}^{\hat{\mu} \hat{\nu}}=\sum_{N_{X}=0}^{\infty} d\left(N_{X}\right) \cdot \bar{\psi}_{\mathbf{s}^{\prime}} q_{\hat{\rho}} \Gamma^{\hat{\rho}} \psi_{\mathbf{s}} \frac{\eta^{\hat{\mu} \hat{\nu}}}{q^{2}+M_{N_{X}}^{2}}
$$

This result can be understood as the sum of the propagators of the fields which satisfy some non-local field equations. The different GSO projection for type IIA and type IIB theories is corresponding to take the different chirality for $\mathbf{s}^{\prime}$ and $\mathbf{s}$. Not all the NS-R fermionic states in type IIA and type IIB string theories in the flat space-time are included in this propagator, but all the NS-R massless fermionic states are included. 
To do the same in the fivebrane background, we need the explicit expression of the Dinstanton state for the bosonic sector in the fivebrane background.

The D-brane boundary state in the $\mathrm{SU}(2)_{k}$ WZW model is discussed in Refs. 17, 18]. It is possible to explicitly construct the D-instanton state. The place where the edge of the open string is fixed is the north or south pole of $S^{3}$. The D-instanton state is the Cardy's state

$$
|C\rangle=\sum_{l=0}^{k} \sqrt{S_{0}}|l\rangle
$$

where

$$
S_{l^{\prime}}^{l}=\sqrt{\frac{2}{k+2}} \sin \left(\pi \frac{\left(l^{\prime}+1\right)(l+1)}{k+2}\right)
$$

and $|l\rangle$ is the Ishibashi state which satisfies

$$
\left\langle l^{\prime}\left|q^{\frac{1}{2}\left(L_{0}^{\mathrm{WZW}}+\tilde{L}_{0}^{\mathrm{WZW}}\right)}\right| l\right\rangle=\delta_{l^{\prime} l} q^{-S_{l, k}} \chi_{l}^{(k)}(q)
$$

with $S_{l, k}=((l+1) / 2)^{2} /(k+2)-3 / 24$ and the $\mathrm{SU}(2)_{k}$ character $\chi_{l}^{(k)}(q)$. In the state $|C\rangle$ there is no explicit dependence on the spatial point where the edge of the open string is fixed. This is due to the fact that the state is constructed in the $\mathrm{SU}(2)_{k}$ invariant way.

The D-brane boundary state in the linear-dilaton background is investigated in Refs. 19 , 20]. It is impossible to impose Dirichlet boundary condition in conformal invariant way to the Feigin-Fuchs field because of the non-zero background charge $Q[19]$. Although we can construct the boundary state which satisfies Dirichlet boundary condition in the same way as Eq. (15), it is not conformal invariant. The Dirichlet boundary state can be defined, not directly applying the Dirichlet boundary condition, but using the Cardy's condition [20]. The states are described in the similar way of Eq.(26):

$$
\left|B_{\mathrm{FF}}(y)\right\rangle=\int \frac{d p}{2 \pi} e^{-i p y}|p\rangle_{\mathrm{FF}},
$$

where

$$
{ }_{\mathrm{FF}}\left\langle p^{\prime}\left|q^{L_{0}^{\mathrm{FF}}+\tilde{L}_{0}^{\mathrm{FF}}-\frac{c_{\mathrm{FF}}}{12}}\right| p\right\rangle_{\mathrm{FF}}=\delta\left(p^{\prime}-p\right) q^{p^{2}} q^{-1 / 12} \prod_{n=1}^{\infty}\left(1-q^{2 n}\right)^{-1}
$$

with $c_{\mathrm{FF}}=1+6 Q^{2} / \alpha^{\prime}$. The states $|p\rangle_{\mathrm{FF}}$ with a continuous parameter $-\infty<p<\infty$ are the highest weight states of the continuous representation of the linear-dilaton conformal field theory with weight $\frac{\alpha^{\prime}}{4}\left(p^{2}+\left(Q / \alpha^{\prime}\right)^{2}\right)$. The boundary state $|B(y)\rangle_{\mathrm{FF}}$ satisfies the Cardy's condition.

$$
\left\langle B_{\mathrm{FF}}(y)\left|q^{L_{0}^{\mathrm{FF}}+\tilde{L}_{0}^{\mathrm{FF}}-\frac{c_{\mathrm{FF}}}{12}}\right| B_{\mathrm{FF}}(y)\right\rangle=\tilde{q}^{-1 / 12} \prod_{n=1}^{\infty}\left(1-\tilde{q}^{2 n}\right)^{-1},
$$


where $\tilde{q} \equiv e^{-2 \pi i / \tau}$ with $q \equiv e^{2 \pi i \tau}$. The parameter $y$ does not necessarily mean the position where the edge of the open string is fixed. But in small $Q$ limit, which is the limit we will consider, it has the meaning of the position. Here, we note that the discrete representation of the linear dilaton conformal field theory need not be considered in the calculation of the tree-level propagator corresponding to the two-point amplitude at genus zero 9$]$.

Using the D-instanton boundary states for the $\mathrm{SU}(2)_{k}$ WZW model and the linear-dilaton theory, we introduce the following boundary state for the calculation of the fermion propagator.

$$
|B(y, \mathbf{s})\rangle^{\hat{\mu}}=\left|B_{X}(y)\right\rangle_{M^{6}} \otimes\left|B_{\mathrm{FF}}(y)\right\rangle \otimes|C\rangle \otimes\left|B_{\mathrm{gh}}\right\rangle \otimes\left|B_{\psi}(\mathbf{s})\right\rangle^{\hat{\mu}}
$$

where $\left|B_{\psi}(\mathbf{s})\right\rangle^{\hat{\mu}}$ is the contribution of the world-sheet fermions in NS-R sector. The state $\left|B_{\psi}(\mathbf{s})\right\rangle^{\hat{\mu}}$ has a little complicated form due to the additional GSO projection.

$$
\left|B_{\psi}(\mathbf{s})\right\rangle^{\hat{\mu}}= \begin{cases}\psi_{-1 / 2}^{\bar{\mu}}|0\rangle_{\mathrm{NS}} \otimes \widetilde{\left|\psi_{\mathbf{s}}\right\rangle_{\mathrm{R}}}, & \left(s_{4}, s_{5}\right)=(1 / 2,-1 / 2),(-1 / 2,1 / 2) \\ \psi_{-1 / 2}^{\mu}|0\rangle_{\mathrm{NS}} \otimes \widetilde{\left|\psi_{\mathbf{s}}\right\rangle_{\mathrm{R}}}, & \left(s_{4}, s_{5}\right)=(1 / 2,1 / 2),(-1 / 2,-1 / 2)\end{cases}
$$

with totally odd number of $s_{i}=-1 / 2$ (odd ten-dimensional chirality) for type IIA theory and with totally even number of $s_{i}=-1 / 2$ (even ten-dimensional chirality) for type IIB theory. The space-time index of the state is restricted depending on the spin state, and the number of the allowed states is the half of the states in case of the flat space-time. This is the realization of the half supersymmetry breaking by the fivebrane background in the world-sheet theory $[9]$.

Now, we can calculate the fermion propagator in the fivebrane background as the transition amplitude between these source boundary states by the propagator operator of Eq.(23) with the operators $L_{0}, \tilde{L}_{0}$ and $\tilde{G}_{0}$ defined in the previous section.

\section{FERMION PROPAGATORS IN FIVEBRANE BACKGROUNDS}

The explicit form of the fermion propagator is described as follows.

$$
\begin{aligned}
P_{F}\left(y^{\prime}-y\right)_{\mathbf{s}^{\prime} \mathbf{s}}^{\hat{\mu} \hat{\nu}} & ={ }^{\hat{\mu}}\left\langle B\left(y^{\prime}, \mathbf{s}^{\prime}\right)\left|D_{F}\right| B(y, \mathbf{s})\right\rangle^{\hat{\nu}} \\
& =\frac{\alpha^{\prime}}{4 \pi} \cdot \frac{1}{2} \int_{|z| \leq 1} d^{2} z \frac{1}{|z|^{2}} \hat{\mu}\left\langle B\left(y^{\prime}, \mathbf{s}^{\prime}\right)\left|\tilde{G}_{0} z^{L_{0}} \bar{z}^{\tilde{L}_{0}}\right| B(y, \mathbf{s})\right\rangle^{\hat{\nu}} .
\end{aligned}
$$

Here,

$$
\begin{aligned}
& L_{0}=L_{0}^{M^{6}}+L_{0}^{\mathrm{FF}}+L_{0}^{W Z W}+L_{0}^{\text {ghost }}-\nu, \\
& \tilde{L}_{0}=\tilde{L}_{0}^{M^{6}}+\tilde{L}_{0}^{\mathrm{FF}}+\tilde{L}_{0}^{W Z W}+\tilde{L}_{0}^{\text {ghost }}-\tilde{\nu}
\end{aligned}
$$


with

$$
\begin{aligned}
L_{0}^{M^{6}} & =\frac{\alpha^{\prime}}{4} p^{\bar{\mu}} p_{\bar{\mu}}+\sum_{n=1}^{\infty} \alpha_{-n}^{\bar{\mu}} \alpha_{\bar{\mu} n}+\sum_{r+\nu=1}^{\infty} r \psi_{-r}^{\bar{\mu}} \psi_{\bar{\mu} r}, \\
L_{0}^{\mathrm{FF}} & =\frac{\alpha^{\prime}}{4}\left(p^{6}-\frac{i Q}{\alpha^{\prime}}\right)^{2}+\frac{\alpha^{\prime}}{4}\left(\frac{Q}{\alpha^{\prime}}\right)^{2}+\sum_{n=1}^{\infty} \alpha_{-n}^{6} \alpha_{n}^{6}+\sum_{r+\nu=1}^{\infty} r \psi_{-r}^{6} \psi_{r}^{6}, \\
L_{0}^{W Z W} & =\left.L_{0}^{W Z W}\right|_{B}+\sum_{r+\nu=1}^{\infty} r \psi_{-r}^{\mu^{\prime}} \psi_{r}^{\mu^{\prime}} \\
L_{0}^{\text {ghost }} & =\sum_{n=1}^{\infty} n\left(b_{-n} c_{n}+c_{-n} b_{n}\right)+\sum_{r+\nu=1}^{\infty} r\left(\beta_{-r} \gamma_{r}-\gamma_{-r} \beta_{r}\right),
\end{aligned}
$$

and the same for $\tilde{L}_{0}$. The eigenvalue of the momentum operator $p^{6}-i Q / \alpha^{\prime}$ corresponds to $p$ in Eq.(29), $\left.L_{0}^{W Z W}\right|_{B}$ is the bosonic contribution, and $\mu^{\prime}=7,8,9$. For NS-R sector, $\nu=1 / 2$ and $\tilde{\nu}=0$. Furthermore,

$$
\tilde{G}_{0}=\tilde{G}_{0}^{M^{6}}+\tilde{G}_{0}^{W_{k}^{(4)}}+\tilde{G}_{0}^{\text {ghost }}
$$

with

$$
\begin{aligned}
\tilde{G}_{0}^{M^{6}} & =-i \sqrt{\frac{\alpha^{\prime}}{2}} p^{\bar{\mu}} \tilde{\psi}_{\bar{\mu} 0}+\text { fermion non-zero modes, } \\
\tilde{G}_{0}^{W_{k}^{(4)}} & =\sqrt{\frac{2}{\alpha^{\prime}}} \sqrt{\frac{2}{k+2}} \tilde{J}_{0}^{\mu^{\prime}} \tilde{\psi}_{0}^{\mu^{\prime}}-i \sqrt{\frac{\alpha^{\prime}}{2}}\left(p^{6}-\frac{i Q}{\alpha^{\prime}}\right) \tilde{\psi}_{0}^{6}+\frac{1}{3 !} \sqrt{\frac{2}{k+2}} \epsilon_{\mu^{\prime} \nu^{\prime} \rho^{\prime}} \tilde{\psi}_{0}^{\mu^{\prime}} \tilde{\psi}_{0}^{\nu^{\prime}} \tilde{\psi}_{0}^{\rho^{\prime}} \\
& + \text { fermion non-zero modes, } \\
\tilde{G}_{0}^{\text {ghost }} & =-\sum_{n=-\infty}^{\infty}\left(\frac{1}{2} n \tilde{\beta}_{-n} \tilde{c}_{n}+2 \tilde{b}_{n} \tilde{\gamma}_{-n}\right) .
\end{aligned}
$$

Here, $\tilde{J}_{0}^{\mu^{\prime}}$ is the zero-mode in the mode expansion of the WZW current $\tilde{J}^{\mu^{\prime}}\left(\tilde{J}_{i}\right.$ with $i=1,2,3$ in Sec correspond to $\tilde{J}^{\mu^{\prime}}$ with $\mu^{\prime}=7,8,9$, respectively). We did not explicitly write the contributions of the non-zero modes of the world-sheet fermions, because they give no contribution to the fermion propagator of Eq.(34).

The calculation is straightforward except for one quantity

$$
\left\langle C\left|\tilde{J}_{0}^{\mu^{\prime}} z^{\left.L_{0}^{\mathrm{WZW}}\right|_{B}} \bar{z}^{\left.\tilde{L}_{0}^{\mathrm{WZW}}\right|_{B}}\right| C\right\rangle
$$

We can show that this quantity vanishes by using the conditions of conformal and $\mathrm{SU}(2)_{k}$ invariance

$$
\begin{aligned}
\left.\tilde{L}_{0}^{\mathrm{WZW}}\right|_{B}|C\rangle & =\left.L_{0}^{\mathrm{WZW}}\right|_{B}|C\rangle, \\
\tilde{J}_{0}^{\mu^{\prime}}|C\rangle & =-J_{0}^{\mu^{\prime}}|C\rangle,
\end{aligned}
$$


and the definition of the Ishibashi state

$$
|l\rangle=\sum_{N, j}|l ; N, j\rangle \otimes U \widehat{|l ; N, j\rangle},
$$

where $U$ is an unitary operator introduced in Ref.[21], and the states $|l ; N, j\rangle$ are in the orthonormal representations of the $\mathrm{SU}(2)_{k}$ Kač-Moody algebra with $0 \leq l \leq k$. The states $|l ; 0, j\rangle$ are in the orthonormal representations of the $\mathrm{SU}(2)$ algebra with $-l \leq j \leq l$, and $N$ denotes the ways of operating some $\tilde{J}_{-n \mu^{\prime}}$ to the states. We obtain

$$
\left\langle C\left|\tilde{J}_{0}^{\mu^{\prime}} z^{\left.L_{0}^{\mathrm{WZW}}\right|_{B}} \bar{z}^{\left.\tilde{L}_{0}^{\mathrm{WZW}}\right|_{B}}\right| C\right\rangle=-\sum_{l, N} S_{0}^{l}|z|^{2 L_{0}(l, N)} \sum_{j}\left\langle l ; N, j\left|J_{0}^{\mu^{\prime}}\right| l ; N, j\right\rangle=0,
$$

where $L_{0}(l, N)$ is the eigenvalue of the operator $\left.L_{0}^{\mathrm{WZW}}\right|_{B}$ on the state $|l ; N, j\rangle$. The second equality comes from the fact that the trace of $J_{0}^{\mu^{\prime}}$ over a representation of $\mathrm{SU}(2)$ is zero.

The result of the calculation is

$$
\begin{aligned}
P_{F}\left(y^{\prime}-y\right)_{\mathbf{s}^{\prime} \mathbf{s}}^{\hat{\mu} \hat{\nu}}= & -i \frac{\sqrt{\alpha^{\prime}}}{2} \int \frac{d^{7} q}{(2 \pi)^{7}} e^{i q_{\tilde{\rho}}\left(y^{\prime}-y\right)^{\tilde{\rho}}} \bar{\psi}_{\mathbf{s}^{\prime}}\left(q_{\tilde{\sigma}} \Gamma^{\tilde{\sigma}}+i \frac{Q}{\alpha^{\prime}} \frac{1}{3 !} \epsilon_{\lambda^{\prime} \kappa^{\prime} \omega^{\prime}} \Gamma^{\lambda^{\prime}} \Gamma^{\kappa^{\prime}} \Gamma^{\omega^{\prime}}\right) \psi_{\mathbf{s}} \eta^{\hat{\mu} \hat{\nu}} \\
& \cdot \sum_{N_{X}=0}^{\infty} d\left(N_{X}\right) \sum_{l=0}^{k} \sum_{m=-\infty}^{\infty}(2(k+2) m+l+1) \sqrt{\frac{2}{k+2}} \sin \left(\pi \frac{l+1}{k+2}\right) \\
& \cdot \frac{1}{q^{\tilde{\delta}} q_{\tilde{\delta}}+\left(\frac{Q}{\alpha^{\prime}}\right)^{2}+M_{N_{X}}^{2}+\frac{4}{\alpha^{\prime}}\left((k+2) m^{2}+(l+1) m\right)},
\end{aligned}
$$

where $\tilde{\rho}, \tilde{\sigma}, \tilde{\delta}=0,1, \cdots, 6$. We used the explicit formula for $\mathrm{SU}(2)_{k}$ character

$$
\chi_{l}^{(k)}(q)=\frac{1}{\eta(\tau)^{3}} \cdot 2(k+2) \sum_{m=\infty}^{\infty}\left(m+\frac{l+1}{2(k+2)}\right) \exp \left(2 \pi i(k+2) \tau\left(m+\frac{l+1}{2(k+2)}\right)^{2}\right),
$$

where $q=e^{2 \pi i \tau}[22]$. In the denominator of the last factor of Eq.(150) the first term is the squared energy-momentum in the flat seven-dimensional space-time, the second term is the universal mass shift due to the linear-dilaton background [23], and the third term is the mass by the usual string excitations $\left(M_{N_{X}}^{2}=4 N_{X} / \alpha^{\prime}\right)$. The fourth term is the contribution which breaks the ten-dimensional space-time interpretation in finite $k$.

The space-time interpretation of $M^{6} \otimes W_{4}^{(k)}$ in this world-sheet theory is possible only in large $k$ limit. In large $k$ limit the contribution of $m=0$ dominates in Eq.(50). The propagator becomes

$$
\begin{aligned}
P_{F}\left(y^{\prime}-y\right)_{\mathbf{s}^{\prime} \mathbf{s}}^{\hat{\mu} \hat{\nu}} \rightarrow & -i \frac{\sqrt{\alpha^{\prime}}}{2} \sum_{N_{X}=0}^{\infty} d\left(N_{X}\right) \int \frac{d^{7} q}{(2 \pi)^{7}} e^{i q_{\tilde{\rho}}\left(y^{\prime}-y\right)^{\tilde{\rho}}} \sum_{l=0}^{k}(l+1) \sqrt{\frac{2}{k+2}} \sin \left(\pi \frac{l+1}{k+2}\right) \\
& \cdot \bar{\psi}_{\mathbf{s}^{\prime}}\left(q_{\tilde{\sigma}} \Gamma^{\tilde{\sigma}}+i \frac{Q}{\alpha^{\prime}} \frac{1}{3 !} \epsilon_{\lambda^{\prime} \kappa^{\prime} \omega^{\prime}} \Gamma^{\lambda^{\prime}} \Gamma^{\kappa^{\prime}} \Gamma^{\omega^{\prime}}\right) \psi_{\mathbf{s}} \eta^{\hat{\mu} \hat{\nu}} \frac{1}{q^{\tilde{\delta}} q_{\tilde{\delta}}+\left(\frac{Q}{\alpha^{\prime}}\right)^{2}+M_{N_{X}}^{2}} .
\end{aligned}
$$


The summation over $l$ is exactly calculable.

$$
\sum_{l=0}^{k}(l+1) \sqrt{\frac{2}{k+2}} \sin \left(\pi \frac{l+1}{k+2}\right)=\sqrt{\frac{k+2}{2}} \cdot \frac{\sin \frac{\pi}{k+2}}{1-\cos \frac{\pi}{k+2}} .
$$

This factor diverges in large $k$ limit as $(\sqrt{k+2})^{3}$, and can be understood as the correspondence of the momentum integration in the $S^{3}$ space with the radius $\alpha^{\prime} / Q=\sqrt{\alpha^{\prime}(k+2)}$. Because in the state $|C\rangle$ there is no explicit spatial point where the edge of the open string is fixed in $S^{3}$, the momentum in $S^{3}$ is not explicitly introduced. The propagation in $S^{3}$ can not be described by using this boundary state. We obtain the propagator in the momentum space as

$$
P_{F}(q)_{\mathbf{s}^{\prime} \mathbf{s}}^{\hat{\mu} \hat{\nu}}=\sum_{N_{X}=0}^{\infty} d\left(N_{X}\right) \bar{\psi}_{\mathbf{s}^{\prime}}\left(q_{\tilde{\sigma}} \Gamma^{\tilde{\sigma}}+i \frac{Q}{\alpha^{\prime}} \frac{1}{3 !} \epsilon_{\lambda^{\prime} \kappa^{\prime} \omega^{\prime}} \Gamma^{\lambda^{\prime}} \Gamma^{\kappa^{\prime}} \Gamma^{\omega^{\prime}}\right) \psi_{\mathbf{s}} \frac{\eta^{\hat{\mu} \hat{\nu}}}{q^{\tilde{\delta}} q_{\tilde{\delta}}+\left(\frac{Q}{\alpha^{\prime}}\right)^{2}+M_{N_{X}}^{2}} .
$$

This result has the similar form to the propagator in the flat space-time, Eq.(25), but it includes some additional contribution which is proportional to three gamma matrices. It would be interesting if the term could result some interesting phenomena like fermion pair condensation, but it is not the case. We have to do further the additional GSO projection. As can be seen in Eq.(33), the additional GSO projection on the spinor index can be described as the six-dimensional chirality projection. The polarization spinor $\psi_{\mathbf{s}}$ should satisfy appropriate chirality conditions. The final result can be described as follows not explicitly including $\psi_{\mathbf{s}}$, but using projection operators.

$$
P_{F}(q)^{\hat{\mu} \hat{\nu}}=\left\{\begin{array}{l}
\sum_{N_{X}=0}^{\infty} d\left(N_{X}\right) \cdot \frac{1 \pm \Gamma_{11}}{2} \cdot \frac{1 \mp \gamma_{6}}{2} \cdot \frac{q_{\bar{\sigma}} \Gamma^{\bar{\sigma}} \eta^{\bar{\mu} \bar{\nu}}}{q^{\tilde{\delta}} q_{\tilde{\delta}}+\left(\frac{Q}{\alpha^{\prime}}\right)^{2}+M_{N_{X}}^{2}} \cdot \frac{1 \pm \gamma_{6}}{2} \cdot \frac{1 \mp \Gamma_{11}}{2} \\
\sum_{N_{X}=0}^{\infty} d\left(N_{X}\right) \cdot \frac{1 \pm \Gamma_{11}}{2} \cdot \frac{1 \pm \gamma_{6}}{2} \cdot \frac{q_{\bar{\sigma}} \Gamma^{\bar{\sigma}} \eta^{\mu \nu}}{q^{\tilde{\delta}} q_{\tilde{\delta}}+\left(\frac{Q}{\alpha^{\prime}}\right)^{2}+M_{N_{X}}^{2}} \cdot \frac{1 \mp \gamma_{6}}{2} \cdot \frac{1 \mp \Gamma_{11}}{2}
\end{array}\right.
$$

for type IIA and type IIB theories, respectively in sign, where $\gamma_{6} \equiv \Gamma^{0} \Gamma^{1} \cdots \Gamma^{5}$ and $\Gamma_{11}=$ $\Gamma^{0} \Gamma^{1} \cdots \Gamma^{9}$.

The lightest $\left(N_{X}=0\right)$ propagation modes can be understood as the components of the supermultiplets in six-dimensional $N=(2,0)$ and $N=(1,1)$ supergravity theories for type IIA and type IIB theories, respectively. Note that there are no propagators of the fields in $\mathrm{SO}(4)$ (local Lorentz) spinor representations with $\Gamma^{\mu}(\mu=6,7,8,9)$ in the numerator, which is consistent with the arguments on the fermion zero-modes in the fivebrane background of low-energy supergravity theories. Namely, the existence of four fermion zero-modes in 
non-trivial four-dimensional space $W_{k}^{(4)}$ means that there are no propagators (or two point functions) in $\mathrm{SO}(4)$ spinor representations, if we believe the path integral formalism for quantization.

In case of type IIA (IIB) theory and $N_{X}=0$, the propagator of the first line of Eq.(55) corresponds to the propagations of one complex six-dimensional spin-3/2 gravitino and one complex six-dimensional spin-1/2 dilatino in the $N=(2,0)(N=(1,1))$ supergravity multiplet. Another pair of the gravitino and dilatino in the supergravity multiplet should be supplied from the R-NS sector. The propagator of the second line of Eq.(55) corresponds to the propagations of four complex spin-1/2 spinor fields in two $N=(2,0)$ tensor multiplets (two $N=(1,1)$ vector multiplets) of the supergravity theory. The R-NS sector gives the fermion components of other two $N=(2,0)$ tensor multiplets (two $N=(1,1)$ vector multiplets).

Note that the obtained propagator correctly describes the propagation at around a certain distance from the place of the fivebrane. The magnitude of the value of the dilaton field becomes large near and far away from the place of the fivebrane (see Eq.(2) with $e^{2 \Phi_{0}}=0$ and $n=k+2$ ), and the perturbative calculation on the string coupling (the genus expansion) does not give correct results. Therefore, the six-dimensional system mentioned in the previous paragraph is not the one confined in the fivebrane, but the system of the bulk with the distance around $\sqrt{\alpha^{\prime}(k+2)}$ away from the place of the fivebrane.

\section{CONCLUSIONS}

We have calculated the fermion propagators in the fivebrane background of type IIA and type IIB string theories. The world-sheet conformal field theory is the combination of the $\mathrm{SU}(2)_{k}$ WZW model and linear-dilaton theory, which describes the non-trivial fourdimensional space, and the theory corresponding to the six-dimensional flat Minkowski space-time. The Kač-Moody level $k$ has been taken even and large number for the tendimensional space-time interpretation. The tree-level calculation is effective at the distance around $\sqrt{\alpha^{\prime}(k+2)}$ away from the place of the fivebrane, where the magnitude of the value of the dilaton field is small.

The form of the obtained propagator is simple, and the lightest propagating modes can be understood as the components of the supermultiplets in six-dimensional $N=2$ supergravity 
theories. The fact that there are no propagations of the fields in $\mathrm{SO}$ (4) (local Lorentz) spinor representations is consistent with the arguments on the fermion zero-modes in low-energy supergravity theories. No signature of the fermion pair condensation has found, which is also consistent with the arguments on the fermion zero-modes in low-energy effective theories.

It might be interesting to attempt the similar calculation in the heterotic string theory. From the arguments on the fermion zero-modes in the low-energy supergravity theory, the pair condensations of gravitino, dilatino and gaugino are expected. Although it is difficult to construct the source boundary state for gravitino and dilatino without destroying Dirichlet boundary condition, it is possible to construct the appropriate boundary state for gaugino. The formation of the gaugino condensation in the low-energy supergravity theory can be understood as the instanton effect at the semi-classical level. It might be interesting to ask the question how the semi-classical effect in the low-energy effective theory is described in the string world-sheet theory.

\section{Acknowledgments}

I would like to thank S. Saito and S.V. Ketov for useful comments. I would specially thank to B.-Y. Hou and R.-H. Yue for valuable discussions and kind hospitality during my stay in Xibei university.

[1] E. Witten, Nucl. Phys. B188, 513 (1981); Commun. Math. Phys. 100, 197 (1985).

[2] K. Konishi, N. Magnoli and H. Panagopoulos, Nucl. Phys. B309, 201 (1988); B323, 441 (1989).

[3] S.B. Giddings and A. Strominger, Nucl. Phys. B306, 890 (1988).

[4] S. Coleman, Nucl. Phys. B307, 867 (1988).

[5] S.-J. Rey, UCSB-TH-89/49, Invited talk given at Workshop on Superstrings and Particle Theory, Tuscaloosa, Alabama, Nov 8-11, 1989; Phys. Rev. D43, 526 (1991).

[6] C.G. Callan, J.A. Harvey and A. Strominger, Nucl. Phys. B359, 611 (1991).

[7] C.G. Callan, J.A. Harvey and A. Strominger, Nucl. Phys. B367, 60 (1991).

[8] S.-J. Rey, SLAC-PUB-5659, Presented at Particle and Fields '91 Conf., Vancouver, Canada, Aug 18-22, 1991. 
[9] I. Antoniadis, S. Ferrara and C. Kounnas, Nucl. Phys. B421, 343 (1994).

[10] V.Ya. Fainberg and A.V. Marshakov, Phys. Lett. B211, 81 (1988).

[11] A.V. Marshakov, Nucl. Phys. B312, 178 (1989).

[12] N. Kitazawa, Mod. Phys. Lett. A17, 2617 (2002).

[13] Y. Katagiri and N. Kitazawa, hep-th/0208069.

[14] A. Cohen, G. Moore, P. Nelson and J. Polchinski, Nucl. Phys. B267, 143 (1986).

[15] P. Di Vecchia, M. Frau, I. Pesando, S. Sciuto, A. Lerda and R. Russo, Nucl. Phys. B507, 259 (2997).

[16] J. Polchinski, "String Theory" Vol. I and II, Cambridge Univ. Pr. (1998).

[17] A. Yu. Alekseev and V. Schomerus, Phys. Rev. D60, 061901 (1999).

[18] G. Felder, J. Fröhlich, J. Fuchs and C. Schweigert, J. Geom. Phys. 34 (2000) 162.

[19] M. Li, Phys. Rev. D54, 1644 (1996).

[20] A. Rajaraman and M. Rozali, JHEP 9912:005 (1999).

[21] N. Ishibashi, Mod. Phys. Lett. A4, 251 (1989).

[22] D. Gepner and E. Witten, Nucl. Phys. B278, 493 (1986).

[23] E. Kiritsis and C. Kounnas, Nucl. Phys. B456, 699 (1995); Nucl. Phys. B422, 472 (1995). 